«Critical incidence reporting» (CIRS) als Qualitätsinstrument bedeutet auch die Einführung einer neuen Fehlerkultur. Die Forderung, solche Meldesysteme flächendeckend zu schaffen, ist legitim. Aber ohne gesetzlichen Rahmen haben diese Instrumente wenig Chancen, dem dafür geschaffenen Zweck zu dienen: Zwischenfälle zu vermeiden, die die Patienten gefährden. Nur mit der notwendigen Vertraulichkeit und Anonymität werden die psychologisch oft belastenden Ereignisse gemeldet. Ziel ist eine Sicherheitskul- tur, die auf Vertrauen basiert. In Anbetracht von möglichen Haftpflichtverfahren ist die Forderung nach einer gesetzlichen Vertraulichkeitsregelung zentral; hier kann das Parlament mit einer entsprechenden Regelung einen wichtigen Beitrag leisten. Die FMH wird diesen Prozess aktiv vorantreiben.

Daniel Herren, Mitglied des Zentralvorstands, Verantwortlicher Ressort DDQ

\title{
10 Jahre Warten auf gesetzliche Zweckbindungsgarantie für CIRS
}

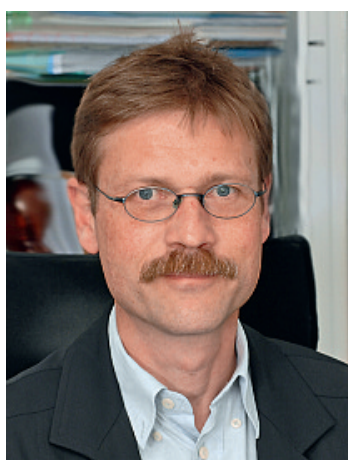

Hanspeter Kuhn
Vor zehn Jahren hat das National Institute of Health (NIH) der USA den Bericht «To err is human» veröffentlicht [1]. Die Idee, dass der Mensch immer perfekt funktioniert, ist eine Illusion. Wer Sicherheit wirksam fördern will, gestaltet deshalb die Abläufe und Systeme so, dass menschliche und organisatorische Pannen möglichst keinen grossen Schaden anrichten. Dabei ist CIRS eine wichtige Ergänzung der übrigen Qualitätssicherungsprogramme: Durch das Melden und Auswerten der kritischen Ereignisse - der «BeinaheSchadenfälle», bei denen es gerade noch mal gut gegangen ist - lassen sich Schwachstellen erkennen und Behandlungsabläufe sicherer machen.

Kritische Ereignisse werden nur gemeldet, wenn im Spital, in der Arztpraxis und in der Fachgesellschaft ein angstfreies Klima besteht. Es braucht eine klare Zweckbindungsgarantie für CIRS und andere Qualitätssicherungsmassnahmen in einem formellen Gesetz, damit die Meldungen und Auswertungen zuverlässig geschützt sind. Der Bericht des NIH hat dies klar gemacht: «Congress should pass legislation to extend [...] protections to data related to patient safety and quality improvement.» [1] Die FMH hat 2001 darauf hingewiesen und einen ausformulierten Vorschlag für die Ergänzung des Allgemeinen Teils des Sozialversicherungsrechts ATSG vorgelegt [2].

Ohne klare gesetzliche Regeln bleibt das Risiko, dass der Richter in einem Zivil- oder Strafverfahren CIRSUnterlagen herausverlangt oder die Personen, die im CIRS gemeldet oder ausgewertet haben, als Zeugen einvernimmt. Dass Swissmedic gegen ihren Willen Medikamentenregistrierungsunterlagen an die Staatsanwaltschaft herausgeben musste, weil das Heilmittelgesetz keine klare Zweckbindungsgarantie dieser Daten beinhaltete [3], sollte als Warnung genügen. Mit organisatorischen Massnahmen - insbesondere Anonymisierung und Vernichtung der Originalmeldung nach der Auswertung - kann das rechtliche Risiko verringert, aber nicht vollständig verhindert werden [4].

Der US-Kongress hat 2005 die nötigen gesetzlichen Grundlagen für CIRS in der Medizin geschaffen [5]. In der Schweiz hat das Parlament im selben Jahr eine Bestimmung im Bundesgesetz über die Luftfahrt ergänzt [6]. Krankenpflegende und Ärzte warten hingegen immer noch auf die gesetzliche Grundlage. Hoffen wir, dass Bundesrat und Parlament aktiv werden, bevor sich bei uns im Gesundheitswesen wiederholt, was dem Luftfahrt-CIRS eines anderen Landes passiert ist: «As an example to all systems, one country's first system was completely destroyed due to lack of reporting after a breach of a reporter's identity.» [7]

Hanspeter Kuhn, stv. Generalsekretär FMH

1 Committee on Quality of Health Care in America. In: Kohn LT, et al. To Err is Human. Building a Safer Health System. Washington, D.C.: Institute of Medicine (National Academy Press); 2000; S. 129.

2 Kuhn HP. «Congress should pass legislation to extend protection». Schweiz Ärztezeitung. 2001;82(26):1394-403. Der deutsche Medizinrechtsprofessor K. Ulsenheimer kommt für Deutschland zum gleichen Schluss: Mildenberger D, Ulsenheimer K. Incident Reporting für ein produktives Riskmanagement. Das Krankenhaus. 2003;(7):1-5.

3 Entscheid des Bundesstrafgerichts vom 15. Februar 2006 , BV.2005.35.

4 Kuhn HP, von Below G. Melden Sie keine Flugzeugunfälle auf diesem Formular. Schweiz Ärztezeitung. 2003;84(26):1399-407.

5 Patient Safety and Quality Improvement Act of 2005 (July 29, 2005).

6 Revision vom 16. Dezember 2005

7 Statement von Linda J. Connell, Director, NASA Aviation Safety Reporting System, vor dem US Subcommittee on Health, Committee on Ways and Means, House of Representatives, February 10, 2000. 